\title{
Smart Control of Automatic Voltage Regulators using K-means Clustering
}

Brook Abegaz

babegaz@luc.edu

J. Kueber

Follow this and additional works at: https://ecommons.luc.edu/engineering_facpubs

Part of the Computer Engineering Commons

\section{Recommended Citation}

Abegaz, Brook and Kueber, J., "Smart Control of Automatic Voltage Regulators using K-means Clustering" (2019). Engineering Science Faculty Publications. 5.

https://ecommons.luc.edu/engineering_facpubs/5

This Conference Proceeding is brought to you for free and open access by the Faculty Publications and Other Works by Department at Loyola eCommons. It has been accepted for inclusion in Engineering Science Faculty Publications by an authorized administrator of Loyola eCommons. For more information, please contact ecommons@luc.edu.

\section{(c) (†) $\ominus$}

This work is licensed under a Creative Commons Attribution-Noncommercial-No Derivative Works 3.0 License. (C) 2019 IEEE. 


\title{
Smart Control of Automatic Voltage Regulators using K-means Clustering
}

\author{
B. Abegaz, Member, IEEE, J. Kueber, Student Member, IEEE
}

\begin{abstract}
The future cyber physical systems consist of voltage regulators distributed across wide geographical areas. In this paper, a smart control approach of voltage regulators is presented for cyber physical system applications. The approach is implemented using K-means clustering algorithms that use data from voltage and current sensors, compute the correlation of changes across the regulators and generate a proportional feedback. Advanced estimation methods are used in cases where the data from the sensors was not available. The results show that the approach could be used to improve the performance of networked, power dependent systems by $94.5 \%$ in terms of overshoot and $9.52 \%$ in terms of response time as compared to other methods of controlling voltage regulators.
\end{abstract}

Index Terms-Cyber-Physical Systems, Voltage Regulators, Automatic Voltage Regulator (AVR) Control, Clustering

\section{INTRODUCTION}

A VOLTAGE REGULATOR provides a stable dc operating voltage regardless of variations on load current, line voltage, frequency, temperature and other related factors. Voltage regulators are essential components for almost all electronic devices. Without voltage regulators, it would be impossible to safely operate or produce electronic systems of all classifications [1].

Voltage regulation has always been a necessary component in power grids. In recent years, the increasing development and implementation of renewable energy sources has created new challenges. Power from distributed generation can flow from the load back to the source, thus creating a bidirectional flow of power [2]. Additionally, unpredictable environmental conditions can also create voltage deficiencies. Modern voltage regulation systems must not only compensate for consumer behavior, but be able to handle various environmental factors as well. Automatic voltage regulator (AVR) systems are used to maintain voltage quality in power grids. A major focus of modern literature is optimization methods for proportional integral derivative (PID) controllers in AVR systems. These AVR systems are composed of amplifier, exciter, generator, and feedback controller blocks.

The need for smart voltage regulation systems was highlighted by an Internal Revenue Service (IRS) outage in 2016. Tax return processing was interrupted for approximately 30 hours when both the primary and backup voltage regulators experienced a hardware failure on the computer server that was responsible for processing millions of Americans' tax returns. The IRS attributed the hardware failures to high-stress conditions. These conditions were likely the result of rapid changes in load voltage [3]. The implementation of a smart voltage regulator could have prevented the outage by adapting to these dynamic load conditions.

Additionally, the U.S. Navy's $\$ 13$ billion carrier, named the USS Gerald R. Ford, experienced a series of voltage regulator malfunctions in its four main turbine generators in 2016. As a result, a major renovation was required for severe damage on one of the turbines [4]. The costly repairs may have been avoided if more advanced voltage regulator systems were implemented.

This article provides an overview of recent developments in smart voltage regulator design, a comparison of different smart control techniques for AVR systems, and proposes a K-means clustering based controller for an AVR system.

\section{Conventional and Smart Voltage Regulation}

Voltage regulation using an AVR has been widely addressed in literature. A control system diagram for an AVR is shown in Figure 1. AVR systems are used in generators where the voltage is too high for circuit-based linear regulators to manage. A controller block is not necessary for functionality and varies with different designs. A commonly used controller is the PID, and a large amount of contemporary literature focuses on optimizing PID parameters in AVR systems. A transfer function G(s) for a PID is given in (1). The proportional gain is $K_{P}$, the integral gain is $K_{I}$, and the derivative gain is $K_{D}$ [5].

$$
G(s)=K_{P}+\frac{K_{I}}{s}+K_{D} s
$$

The amplifier can be either another DC generator or a solidstate amplifier. The function of a first order amplifier is modeled in (2), where $T_{A}$ is the amplifier time constant, $K_{A}$ is the amplifier gain, $V_{R}$ is the exciter input produced by amplifying the difference between the reference voltage and a transducer signal and $V_{I N}$ is the amplifier input. The exciter controls the terminal voltage magnitude of the generator [6]. In some cases, the reference voltage may change due to certain load requirements. A dynamic reference voltage must be considered in smart AVR systems for power grid applications.

$$
T_{A} \frac{d V_{R}}{d t}=-V_{R}+K_{A} V_{I N}
$$




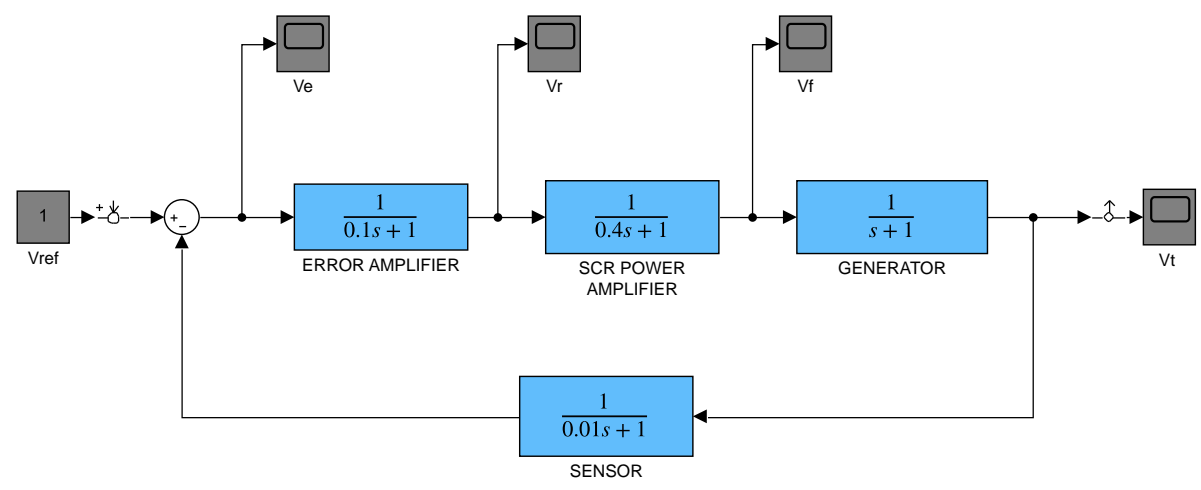

Figure 1: A Conventional Automatic Voltage Regulator (AVR)

Literature on the smart control of voltage regulators proposes techniques that often fall under the following categories: heuristic algorithms, fuzzy logic, and machine learning. Heuristic algorithms are designed to replicate processes observed in nature to solve optimization problems. Fuzzy logic controllers use well defined membership functions to determine the range of an output. Frequently employed machine learning algorithms consist of neural networks and genetic algorithms.

\section{A. The Use of Smart Voltage Regulators}

A notable smart design for an AVR system was proposed in 2009, where a genetic algorithm (GA) was used to obtain optimal PID parameters. The algorithm accurately produced optimal parameters for the PID controller in 72 seconds. Using the optimal gains produced by the GA, the researchers developed a Sugeno fuzzy logic model. This system could produce optimal gains for the PID controller in less than one second for real-time operations and on-line applications [7].

The following year, the same researchers from [7] applied a differential evolution algorithm to find the optimal parameters for AVR controller gains and power system stabilizer variables simultaneously [8]. Although similar to the GA, the new method showed a better performance than the previous work.

In the same year, a self-tuning PID controller using a recursive least-square based linearization and feedback was implemented to find the control system parameters, and well developed algorithms were then used to calculate the optimal gain. The system took 30 seconds to converge to time constants used to establish PID controller gain with a $10 \%$ error on the root mean squared (rms) voltage [9].

In 2011, a PID controller design using a particle swarm optimization (PSO) algorithm was presented. Based on the behavior of social organisms, the PSO algorithm initially generates candidates with an initial position and a velocity. The algorithm tracked the position and the velocity of each particle as it went through the search space to find the maximum or the minimum of the function. Each particle remembered the best value it achieved while the algorithm stored the best value among all of the particles. The algorithm was implemented to determine the optimal three parameters for the PID controller. Simulations demonstrated a fast and efficient search for the optimal PID controller parameters and a step response of 0.2762 seconds, which was superior to the previous GA method. The settling time following this response was 0.4018 seconds [10].
In 2013, a particle swarm optimization (PSO) based tuning of a PID controller was presented [11]. A simplified PSO algorithm and an adaptive PSO (APSO) algorithm were proposed for optimizing PID settings. The simplified PSO algorithm disregarded the swarm's best global position and the particles were chosen randomly for the update. The simplified algorithm allowed for easy tuning of behavioral parameters. In the APSO, the inertial weight of the particles varied according to their best fitness, promoting a more effective exploration that resulted in a faster convergence. The APSO outperformed the previously mentioned methods in both convergence and accuracy, with a settling time of 0.564 seconds and a peak amplitude of 1.01V [11].

In the same year, researchers used Matlab GUI to develop and simulate an AVR with a PID controller. A heuristic method called Ziegler-Nichols was used to tune the parameters of the PID controller. In the method, the integral and the derivative gains were initially set to zero, while the proportional gain was increased until it reached the critical ultimate gain. The output then oscillated at this point. With a rise time over 0.3 seconds, this method was not as efficient as a PSO or a GA. However, the developed GUI simplified the testing process, and was compatible with other tuning methods [12].

Additionally, a hybrid control system for an automatic voltage regulator was proposed for smart grid applications. The design hybridized a fuzzy sliding mode control and a radial bias function network and incorporated a neural network supervised learning procedure. The goal was to improve the stability and the performance of the overall system [13].

Later in 2013, researchers optimized the PID controller using the Taguchi Combined Genetic Algorithm (TCGA). First, an analysis of means was carried out by the Taguchi method to determine optimal values for PID controller parameters. The two most influential design variables were selected by analysis of variance. Then, optimum values for the two influential design variables were found using a multi-objective genetic algorithm. The saturation limit and the proportional, integral, and derivative gains were used to define the search space for the optimization problem. Results of the simulation demonstrated superiority of TCGA in terms of optimized step response of the terminal voltage to GA and PSO methods. TCGA produced a settling time of 0.52 seconds while PSO and GA produced settling times of 0.81 seconds and 0.86 seconds respectively. Furthermore, the maximum percent overshoot 
produced by the TCGA was just $0.36 \%$ of that produced by the PSO [5].

Later in 2017, a fuzzy logic controller based AVR design was presented. The proposed fuzzy logic controller used triangular membership functions, five linguistic variables with twentyfive fuzzy rules, and inputs of error voltage and its derivative. Simulations of the fuzzy logic controller showed a four seconds settling time, which was faster than all of the compared PID controller variants. It also provided lower overshoot as compared to the other controllers, albeit a slower initial response [14].

Methods to determine optimal PID controller parameters for AVR systems are continuing to be developed. A grasshopper optimization algorithm (GOA) was recently presented for this purpose. This algorithm imitated the behavior of grasshoppers, where repulsion forces urged them to move about the search space and attraction forces guided them to promising regions. The results showed that the algorithm outperforms the previously proposed control methods in maximum overshoot, settling time, rise time, and peak time [15].

\section{B. K-means Clustering}

$\mathrm{K}$-means clustering is an unsupervised machine learning algorithm in which data is grouped into clusters based on their similarity. The clusters are mutually exclusive and $K$ represents the number of clusters that were formed. K-means clustering is unique compared to other clustering methods because it relies only on observations rather than hierarchical clustering. Therefore, using K-means clustering is more practical when dealing with large quantities of data.

The controller in this smart AVR system employs a unique $\mathrm{K}$-means clustering algorithm to provide the input to the proportional gain of the PID controller. The squared Euclidean metric shown in (3) was chosen to determine distances for grouping data into clusters. A centroid, or a row vector, is $c$ and $x$ is an observation, or a row of a numeric data matrix [16].

$$
d(x, c)=(x-c)(x-c)^{\prime}
$$

The K-means algorithm is used to initiate the clustering. An observation is selected at random from the data and set as the first centroid $c_{1}$. The distance $d$ from each observation $x_{m}$ to $c_{1}$ is then computed using (3). The next centroid $c_{2}$ is chosen at random with the probability shown in (4). This is repeated until $K$ centroids have been selected from $n$ data points [17].

$$
\frac{d^{2}\left(x_{m}, c_{1}\right)}{\sum_{j=1}^{n} d^{2}\left(x_{j}, c_{1}\right)}
$$

In this paper, a new type of the K-means clustering algorithm is used to group the output of the AVR system into five clusters based on similarity. Five clusters were chosen to group extra low, low, medium, high, and extra high voltages. The error voltage is grouped into one of the 5 clusters and the proportional voltage for the PID is generated based on the center of mass of the grouping. The integral and the derivative gains of the PID were set to 3 and 1 respectively. The integral and the derivative of the error voltage could also be grouped into clusters to generate the integral and derivative gains of the PID controller.

K-means clustering was chosen over other clustering algorithms like hierarchal clustering and density-based spatial clustering of applications with noise (DBSCAN). DBSCAN was not used because it examines the shape of clusters, which is unnecessary for this work. Hierarchal clustering is used to sort data into multi-level cluster trees. Since this work does not involve hierarchal data, this approach was not selected. Kmeans is a relatively simple unsupervised clustering algorithm. It is applicable to this work since it is able to group 2 dimensional data and output a group center of mass.

\section{EXPERIMENTAL SETUP}

Simulations of four smart AVR systems were performed using MATLAB and Simulink. A PID controller block was added in series before the error amplifier shown in Figure 1. The proportional, integral, and derivative gains were set to 8,3 , and 1 respectively. The filter coefficient $N$ was set to 100 . The terminal voltage, error voltage, amplifier voltage, and exciter voltages were measured over a duration of 5 seconds for a fixed reference voltage of $1 \mathrm{~V}$. The reference voltage was then changed to a pulse wave with an amplitude of $1 \mathrm{~V}$ and a period of $1.60 \mathrm{~s}$ and the terminal, error, amplifier, and exciter voltages were measured again.

A Model Predictive Control (MPC) block was added in parallel with the PID controller. The MPC controller was tuned as proposed in [17]. The voltages for this configuration were measured as previously described.

A Fuzzy Logic controller replaced the PID and MPC blocks in the smart AVR system and the voltage profiles were obtained once again. The Fuzzy Logic controller was coded in MATLAB and used 5 triangular membership functions to classify inputs.

Finally, a PID controller was added once again to the system in place of the Fuzzy Logic controller and K-means clusters were used to classify data and generate proportional gains. Kmeans algorithms divided data into 5 categories based on model data generated by the user and updated the proportional gain of the controller according to the terminal voltage.

\section{Results}

The simulations of the PID, MPC, Fuzzy Logic, and Kmeans controlled AVR systems are shown below in Figure 2 through Figure 9. Figure 2 shows the terminal voltages of the smart AVR systems with a fixed reference voltage. TABLE I shows a comparison of the smart AVR systems for a fixed reference voltage. Figure 3 shows the terminal voltages of the smart AVR systems with a dynamic reference voltage. Table II shows a comparison of the smart AVR systems for a dynamic reference voltage. Rise time is the time required for the voltage to rise from $10 \%$ to $90 \%$ of its steady value. Fall time is the time taken for the voltage to fall between the previously specified values. Settling time is the time taken for the voltage to converge within $5 \%$ of the reference voltage $V_{R E F}$. Overshoot is calculated using (5), where $V_{P E A K}$ is the peak voltage value.

$$
O S=\frac{V_{P E A K}-V_{R E F}}{V_{R E F}} \times 100 \%
$$




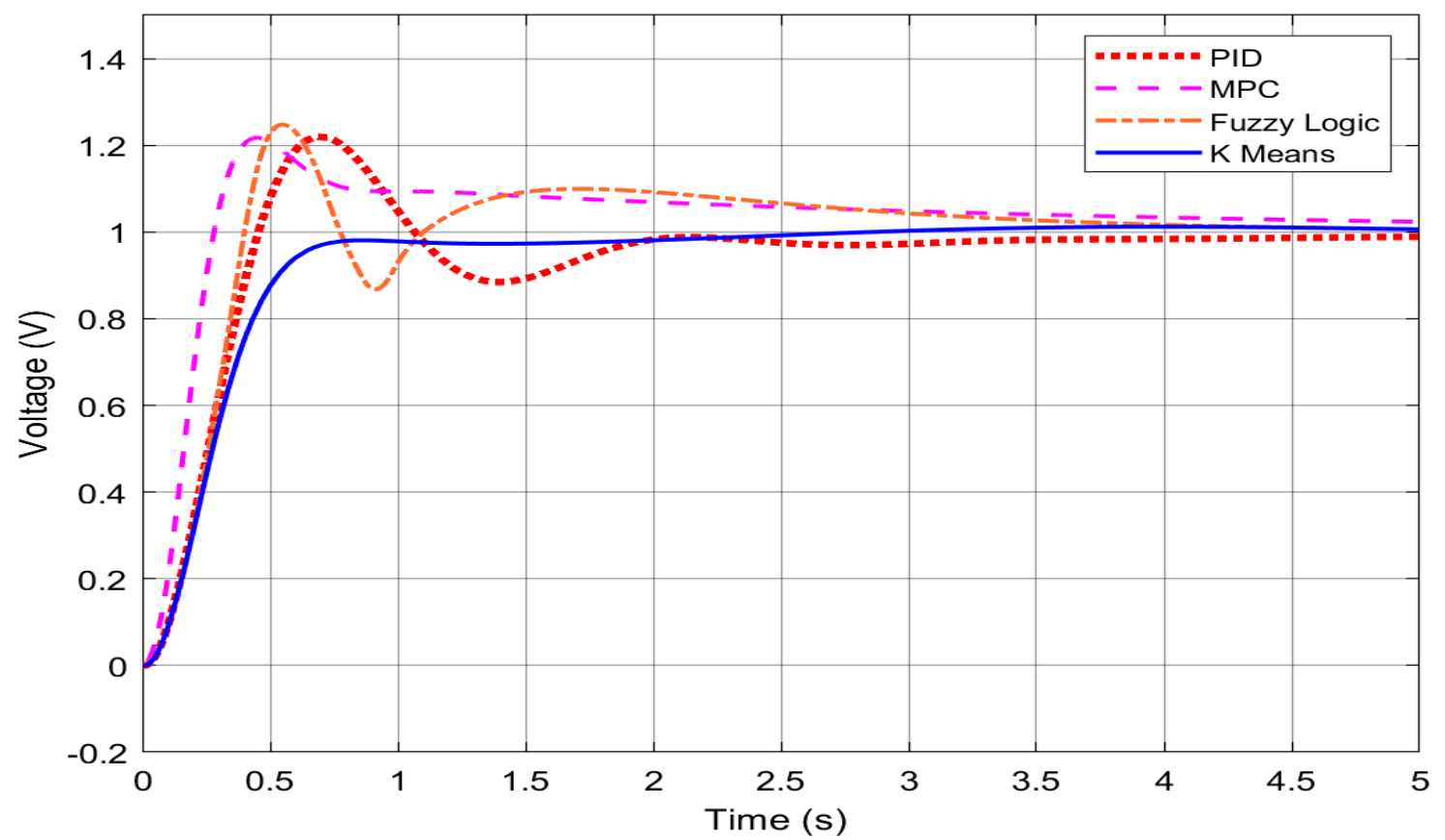

Figure 2: Terminal Voltage Stability Comparison

TABle I: A COMPARISON OF AVR CONTROL METHODS

\begin{tabular}{|c|c|c|c|c|}
\hline Controller & PID & $\begin{array}{c}\text { Model Predictive } \\
\text { Control }\end{array}$ & Fuzzy Logic & K-means \\
\hline Overshoot & $22 \%$ & $21.8 \%$ & $24.8 \%$ & $\mathbf{1 . 2 \%}$ \\
\hline Rise Time & $0.301 \mathrm{~s}$ & $\mathbf{0 . 1 8 2}$ & $0.260 \mathrm{~s}$ & $0.420 \mathrm{~s}$ \\
\hline Settling Time & $1.117 \mathrm{~s}$ & $2.41 \mathrm{~s}$ & $2.28 \mathrm{~s}$ & $\mathbf{0 s}$ \\
\hline Peak Value & $1.220 \mathrm{~V}$ & $1.218 \mathrm{~V}$ & $1.248 \mathrm{~V}$ & $\mathbf{1 . 0 1 2 V}$ \\
\hline
\end{tabular}

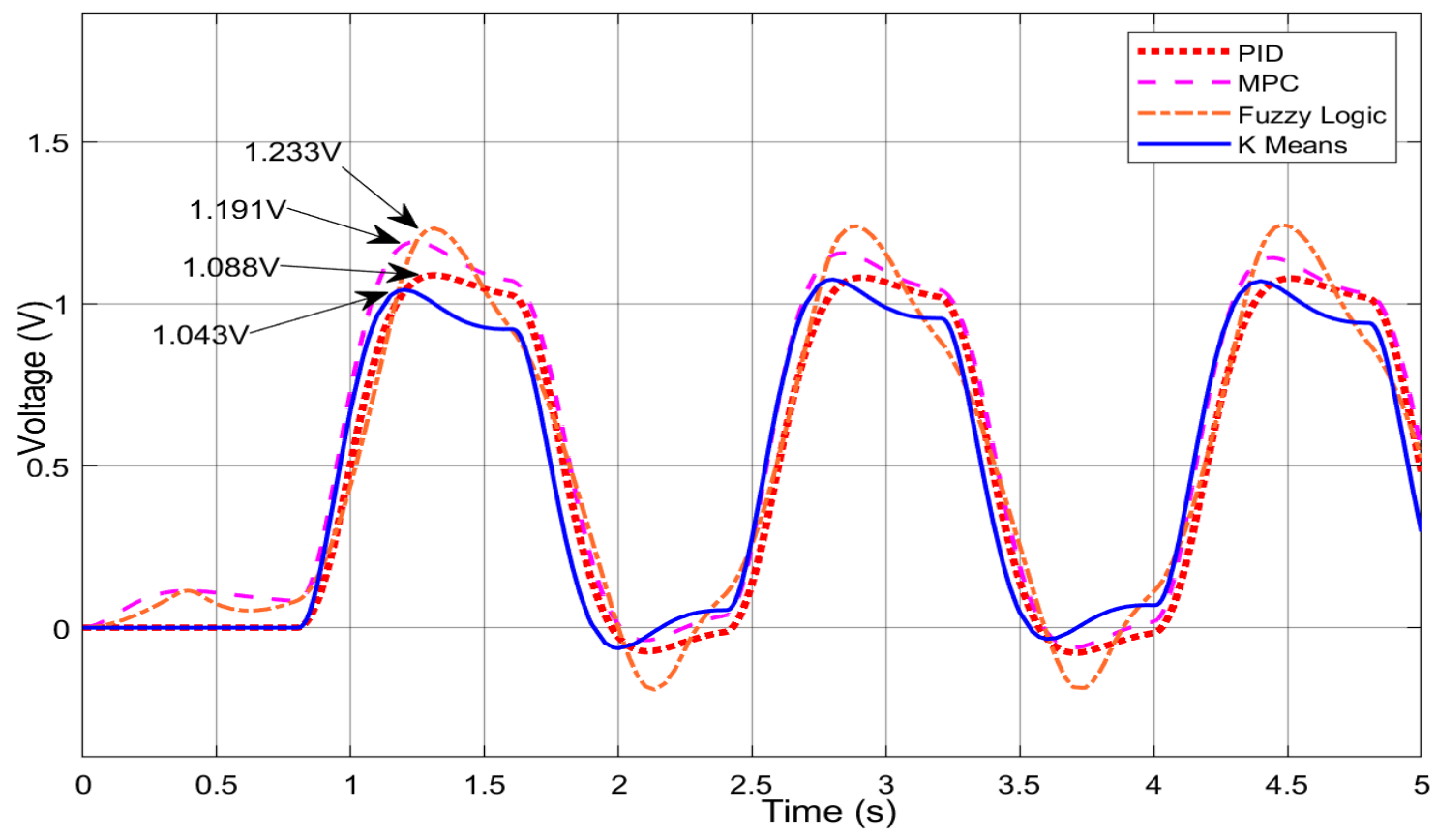

Figure 3: Terminal Voltage Values with Dynamic Reference Voltage 
Table II: A Comparison of AVR Control Methods With a Dynamic ReFEREnCe Voltage

\begin{tabular}{|c|c|c|c|c|}
\hline Controller & PID & $\begin{array}{c}\text { Model Predictive } \\
\text { Control }\end{array}$ & Fuzzy Logic & K-means \\
\hline Overshoot I & $8.8 \%$ & $19.1 \%$ & $23.3 \%$ & $\mathbf{4 . 3 \%}$ \\
\hline Rise Time I & $0.236 \mathrm{~s}$ & $0.212 \mathrm{~s}$ & $0.307 \mathrm{~s}$ & $\mathbf{0 . 2 0 5 s}$ \\
\hline Peak Value I & $1.088 \mathrm{~V}$ & $1.191 \mathrm{~V}$ & $1.233 \mathrm{~V}$ & $\mathbf{1 . 0 4 3 V}$ \\
\hline Fall Time I & $0.23 \mathrm{~s}$ & $0.233 \mathrm{~s}$ & $0.34 \mathrm{~s}$ & $\mathbf{0 . 2 2 4 s}$ \\
\hline Overshoot II & $8.2 \%$ & $15.7 \%$ & $24 \%$ & $\mathbf{7 . 6 \%}$ \\
\hline Rise Time II & $0.228 \mathrm{~s}$ & $\mathbf{0 . 2 s}$ & $0.31 \mathrm{~s}$ & $0.208 \mathrm{~s}$ \\
\hline Peak Value II & $1.082 \mathrm{~V}$ & $1.157 \mathrm{~V}$ & $1.24 \mathrm{~V}$ & $\mathbf{1 . 0 7 6 V}$ \\
\hline Fall Time II & $0.232 \mathrm{~s}$ & $0.231 \mathrm{~s}$ & $0.375 \mathrm{~s}$ & $\mathbf{0 . 2 1 8 s}$ \\
\hline Overshoot III & $7.9 \%$ & $14.2 \%$ & $24.5 \%$ & $\mathbf{7 . 1 \%}$ \\
\hline Rise Time III & $0.229 \mathrm{~s}$ & $\mathbf{0 . 1 9 9} \mathrm{s}$ & $0.318 \mathrm{~s}$ & $0.215 \mathrm{~s}$ \\
\hline Peak Value III & $1.079 \mathrm{~V}$ & $1.142 \mathrm{~V}$ & $1.245 \mathrm{~V}$ & $\mathbf{1 . 0 7 1 V}$ \\
\hline
\end{tabular}

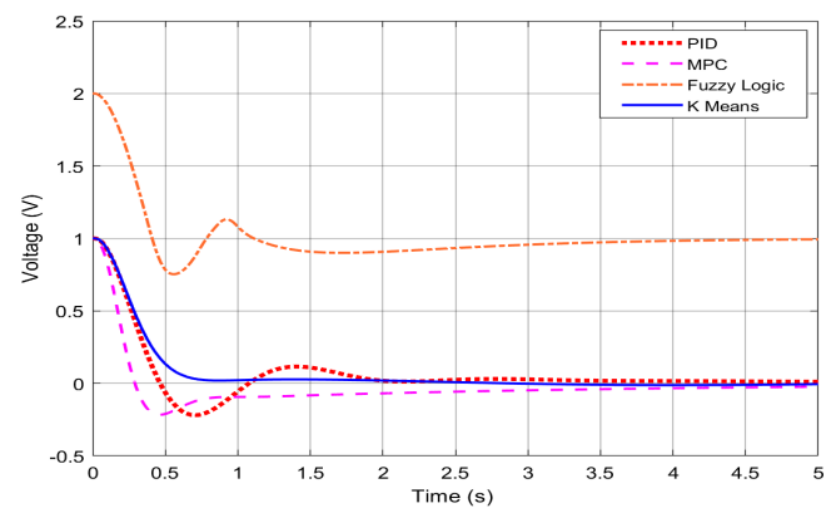

Figure 4: Error Voltage Comparison

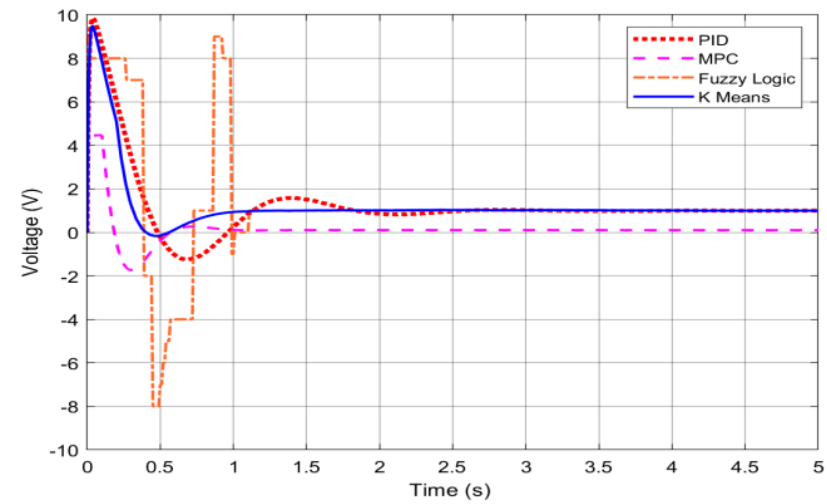

Figure 5: Amplifier Voltage Comparison

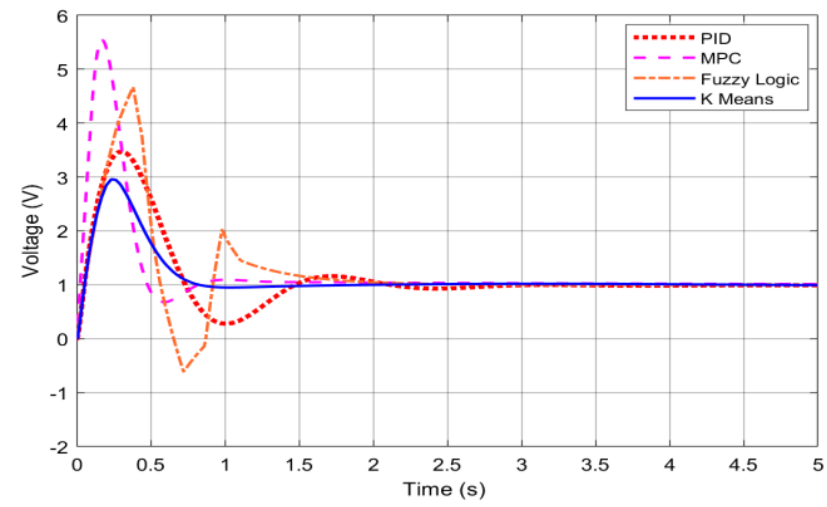

Figure 6: Exciter Voltage Comparison

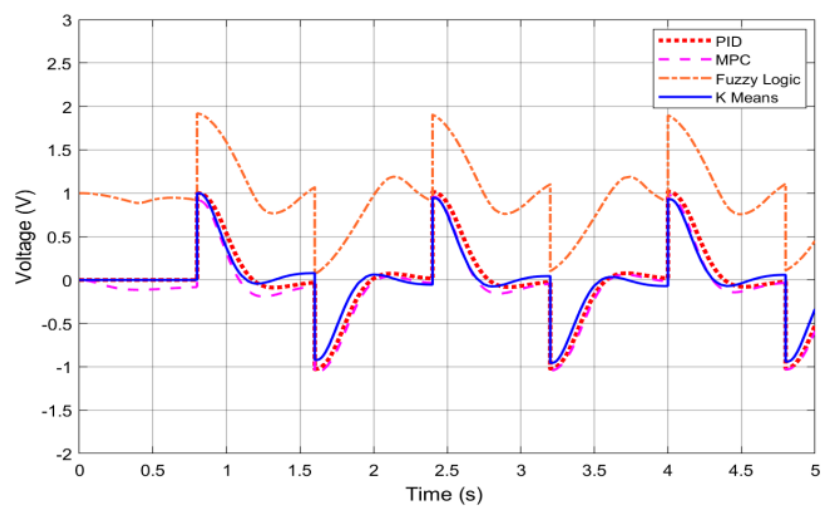

Figure 7: Error Voltage for Dynamic Reference Voltage

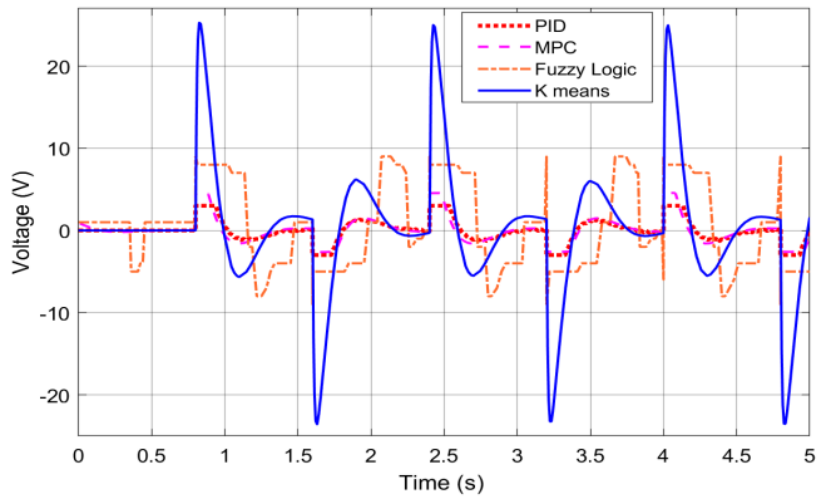

Figure 8: Amplifier Voltage for Dynamic Reference Voltage

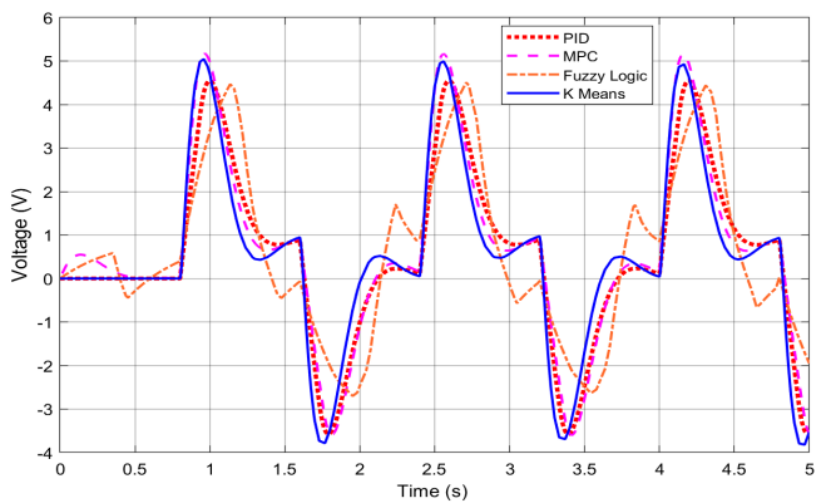

Figure 9: Exciter Voltage for Dynamic Reference Voltage 


\section{DISCUSSION}

The strengths and weaknesses of the different smart controllers for AVR systems are shown in Figure 2 and Table I. The MPC yields the fastest response time of 0.182 seconds. Furthermore, the overshoot produced by the MPC is superior to both the PID and Fuzzy Logic by $0.2 \%$ and $3 \%$ respectively. However, the PID has a faster settling time than the MPC by 1.293 seconds. The K-means controlled AVR system is far superior to the other smart AVR systems in terms of stability. The maximum percent overshoot is only $1.2 \%$ and therefore the settling time is 0 seconds since the peak value is within $5 \%$ of the reference voltage. The downside of the K-means controller is a slow rise time of 0.42 seconds. This rise time is 0.238 seconds slower than the rise time of the MPC controller.

The simulations of smart AVR systems with dynamic reference voltages provides evidence that the rise time of the Kmeans controller can compete with the rise times of the other controllers. The average rise time of the K-means controller over 3 pulse waves is 0.209 seconds. This rise time is only 0.005 seconds slower than that of the MPC and faster than both the PID and Fuzzy Logic controllers by 0.022 seconds and 0.103 seconds respectively. The fall time of the K-means controller is the fastest of the four controllers with an average of 0.221 seconds. The K-means controller continues to perform the best in terms of stability with an average overshoot of $6.33 \%$. These results illustrate a K-means controller is the superior approach for stability in smart AVR systems. Both the K-means and MPC controllers are used in conjunction with the PID controllers. This demonstrates that integrating multiple controllers in a smart AVR system is the correct approach in generating power management solutions.

\section{CONCLUSION}

The AVR control approach presented in this paper is implemented using K-means clustering algorithms that use data from voltage and current sensors, compute the correlation of changes across the regulators and generate a feedback. The results show that the K-means clustering approach could be used to improve the performance of networked, power dependent systems by $94.5 \%$ in terms of overshoot and $9.52 \%$ in terms of response time as compared to other methods of controlling voltage regulators. Future work will address the design and integration of multilevel converter and controller techniques for cyber physical system implementations. Additionally, future research could explore cases where several networked smart AVR systems are implemented. In those cases, terminal voltages of networked smart AVR systems as well as their higher-order derivatives shall be used to generate feedback voltages across the network. It will be useful to calculate systems equations for networked smart AVR systems based on the transfer functions provided in this paper depending on the number of regulators connected in series or parallel.

\section{ACKNOWLEDGMENT}

The authors would like to thank the Office of Research Services (ORS) at Loyola University Chicago.

\section{REFERENCES}

[1] A. P. Brokaw, "Stable Low Dropout Voltage Regulator Controller". United States of America Patent 5686821, 11 Nov 1997.

[2] M. Yalla, "Design of a New Operating Mode for Voltage Regulator Controls in a Smart Distribution System," in IEEE Rural Electric Power Conference, Columbus, OH, 2017.

[3] K. McCoy, "IRS outage caused by back-to-back failures, not cyberattack," USA Today, 12 Feb. 2016. [Online]. Available: https://www.usatoday.com/story/money/2016/02/12/irs-computeroutage-caused-back--back-failures/80303306/. [Accessed 21 Aug. 2018].

[4] MAREX, "Carrier USS Ford Suffers Another Bearing Failure," The Maritime Executive, 9 May 2018. [Online]. Available: https://www.maritime-executive.com/article/carrier-uss-ford-suffersanother-bearing-failure\#gs.hjmsVlk. [Accessed 21 Aug 2018].

[5] H. M. Hasanien, "Design Optimization of PID Controller in Automatic Voltage Regulator System Using Taguchi Combined Genetic Algorithm Method," IEEE Systems Journal, vol. 7, no. 4, pp. 825-31, 2013.

[6] P. W. Sauer, "Power System Dynamics and Stability," Upper Saddle River, New Jersey, Prentice-Hall, 1998, pp. 70-71.

[7] D. Devaraj and B. Selvabala, "Real-Coded Genetic Algorithm and Fuzzy Logic Approach for Real-Rime Runing of Proportional-IntegralDerivative Controller in Automatic Voltage Regulator System," Published in IET Generation, Transmission \& Distribution, vol. 3, no. 7, pp. 641-49, 2009.

[8] B. Selvabala and D. Devaraj, "Co-Ordinated Tuning Of AVR-PSS Using Differential Evolution Algorithm," in 2010 Conference Proceedings IPEC, Singapore, 2010.

[9] K. Kim, P. Rao and J. A. Burnworth, "Self-Tuning of the PID Controller for a Digital Excitation Control System," IEEE Transactions on Industry Applications, vol. 46, no. 4, pp. 1518-24, 2010.

[10] M. S. Rahimian and K. Raahemifar, "Optimal PID Controller Design for AVR System Using Particle Swarm Optimization Algorithm," in 24th Canadian Conference on Electrical and Computer Engineering, Niagara Falls, ON, 2011.

[11] J. F. Nirmal and D. J. Auxillia, "Adaptive PSO based tuning of PID controller for an Automatic Voltage Regulator system," in International Conference on Circuits, Power and Computing Technologies, Nagercoil, India, 2013.

[12] M. B. Bayram, H. İ. Bülbül, C. Can and R. Bayindir, "Matlab/GUI based basic design principles of PID controller in AVR," in 4th International Conference on Power Engineering, Energy and Electrical Drives, Istanbul, Turkey, 2013.

[13] T.-C. Ou, T.-P. Tsao, C.-M. Hong and C.-H. Chen, "Hybrid Control System for Automatic Voltage Regulator in Smart Grid," in International Conference on Machine Learning and Cybernetics, Tianjin, China, 2013.

[14] T. Gupta and D. K. Sambariya, "Optimal Design of Fuzzy Logic Controller for Automatic Voltage Regulator," in International Conference on Information, Communication, Instrumentation and Control, Indore, India, 2017.

[15] B. Hekimoğlu and S. Ekinci, "Grasshopper Optimization Algorithm for Automatic Voltage Regulator System," in 5th International Conference on Electrical and Electronic Engineering, Istanbul, Turkey, 2018.

[16] The MathWorks, Inc, "Documentation," The MathWorks, Inc, 2019. [Online]. $\quad$ Available: https://www.mathworks.com/help/stats/kmeans.html. [Accessed 25 April 2019].

[17] D. Arthur and S. Vassilvitskii, "K-means++: The Advantages of Careful Seeding," in SODA '07: Proceedings of the Eighteenth Annual ACMSIAM Symposium on Discrete Algorithms, New Orleans, Louisiana, 2007.

[18] T. H. M. T. K. Y. M. Qing Liu, "PID-MPC Based Automatic Voltage Regulator Design in Wide-Area Interconnect Power System," International Journal of Emerging Technology and Advanced Engineering, vol. 4, no. 8, pp. 412-17, 2014.

[19] X. Zhang, J. Hodge and M. Attaway, "Intelligent Voltage Regulator in Smart Grid Distribution System," in 2014 China International Conference on Electricity Distribution (CICED 2014), Shenzhen, China, Sep. 2014. 